\title{
Editorial
}

\section{Advances in Mathematical Methods for Image and Signal Processing}

\author{
Feng Gao, ${ }^{1}$ Gongzhu Hu, ${ }^{2}$ Wenying Feng, ${ }^{3}$ Tokuro Matsuo, ${ }^{4}$ and Vangalur Alagar ${ }^{5}$ \\ ${ }^{1}$ Science School, Qingdao Technological University, Qingdao, Shandong 266033, China \\ ${ }^{2}$ Department of Computer Science, Central Michigan University, Mount Pleasant, MI 48859, USA \\ ${ }^{3}$ Department of Computing \& Information Systems, Department of Mathematics, Trent University, Peterborough, \\ ON, Canada K9J $7 B 8$ \\ ${ }^{4}$ Graduate School of Industrial Technology, Advanced Institute of Industrial Technology, Higashi-Oi, Shinagawa 140-0011, Japan \\ ${ }^{5}$ Department of Computer Science and Software Engineering, Concordia University, Montreal, QC, Canada H4B 1R6
}

Correspondence should be addressed to Feng Gao; gaofeng99@sina.com

Received 28 May 2014; Accepted 28 May 2014; Published 16 June 2014

Copyright (C) 2014 Feng Gao et al. This is an open access article distributed under the Creative Commons Attribution License, which permits unrestricted use, distribution, and reproduction in any medium, provided the original work is properly cited.

Mathematical methods for signal and image processing present the mathematical methodology for generic image and signal analysis tasks. The existing literature on image methodology is rather scattered. This special issue brings together some seemingly different points of view to stress their conceptual relations and analogies. In this issue, some papers focus on specific applications, while some other papers discuss the methodological frameworks on which specific applications are built. Covering many topics at the forefront of current research, this issue will be of particular interest to researchers in the fields of computer vision, image processing, signal processing, medical imaging, visual perception, pattern recognition, and so on.

Among the 16 exciting papers compiled in this special issue, two papers discuss fundamental mathematical concepts. J.-J. Yao and Z.-L. Jiang consider the skew circulant and skew left circulant matrices with continuous Lucas numbers. They discuss the invertibility of the skew circulant matrices and present the determinant and the inverse matrices by constructing the transformation matrices. H.-B. Tu et al. focus on the problem of self-occlusion in the field of human action recognition. In their paper, a new adaptive occlusion state behavior recognition approach is presented based on Markov random field and probabilistic latent semantic analysis.

Two papers present the specific applications of mathematical methods in image and signal processing. W. Liu et al. propose a new vibration signal analysis approach to detect the coal-gangue interface based on singular value decomposition (SVD) techniques and support vector machines (SVMs). The empirical mode decomposition (EMD) is also used in their work. G. Hua and D. Jiang propose a new self-adaptive method for image denoising based on visual characteristics.

One paper deals with high speed video images. T. Koç and T. Çiloğlu propose an automatic method for segmenting glottis in high speed endoscopic video (HSV) images of vocal folds. Their method is based on image histogram modeling.

In the area of image encryption and image coding, S. Zhang et al. propose a joint encryption and reversible data hiding scheme. R. Gomathi and A. Vincent Antony Kumar introduce a new framework for image coding that uses image inpainting method. In the proposed algorithm, the input image is subjected to image analysis to remove some of the portions purposefully.

There are two papers in the area of communication signal processing. J. Wang et al. discuss $60 \mathrm{GHz}$ wireless communications over the additive white Gaussian noise channel. Channel capacity with quadrature amplitude modulation (QAM) is investigated for the unlicensed $59-64 \mathrm{GHz}$ radio spectrum set aside by FCC. Their results prove that QAM is an attractive scheme for $60 \mathrm{GHz}$ wireless communications. D. Yumin and X. Shufen propose quantum random walk optimization model and algorithm in network cluster server traffic control and task scheduling. 
Two papers deal with image retrieval and image matching. M. Xiao et al. propose new optimization strategies on vocabulary tree building, retrieval, and matching methods. F. Nayyeri and M. F. Nasrudin study image matching by using dimensionally reduced embedded earth mover's distance.

In the area of image recognition and image reconstruction, M. A. Shayegan et al. propose a new two-stage approach for dimensionality reduction. Their method is based on onedimensional and two-dimensional spectrum diagrams of standard deviation and minimum to maximum distributions for initial feature vector elements. Y. Luo et al. study exact CS reconstruction condition of undersampled spectrum-sparse signals. They mathematically prove that, in certain cases, the exact CS reconstruction of a spectrum-sparse signal from undersampled data is impossible.

Three papers focus on classical mathematical methods of image processing. B. Subramanian et al. discuss the properties of wavelets and multiwavelets with regard to the denoising capability compared to conventional filtering techniques. L. $\mathrm{Xu}$ et al. propose a novel algorithm for image sequence fusion and denoising simultaneously in 3D shearlet transform domain. C. Chi and F. Gao propose an algorithm based on fractional difference for the edge extraction of thenar palm print image.

By compiling these papers, we hope to enrich our readers and researchers with respect to the advances in mathematical methods for image and signal processing.

Feng Gao

Gongzhu $\mathrm{Hu}$

Wenying Feng

Tokuro Matsuo

Vangalur Alagar 


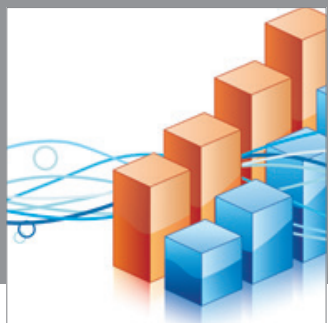

Advances in

Operations Research

mansans

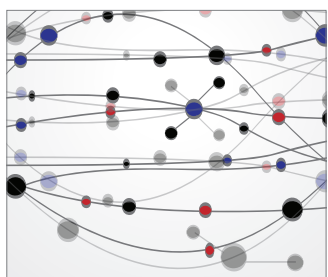

The Scientific World Journal
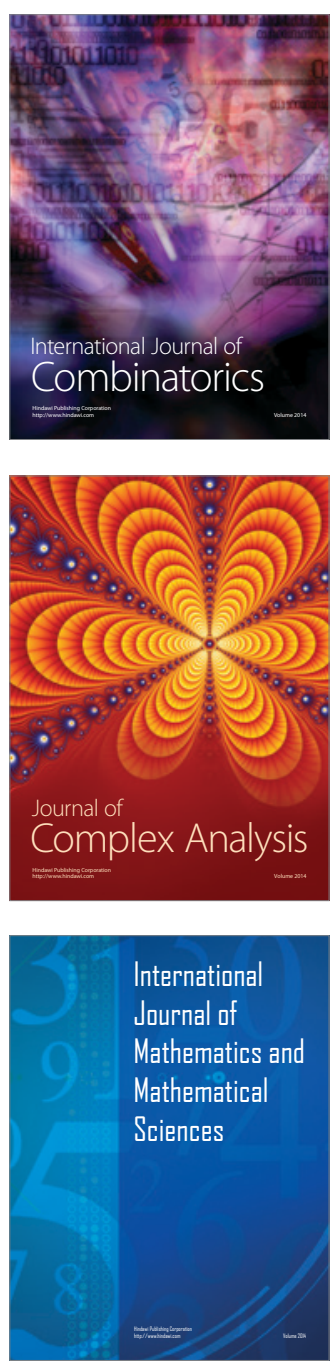
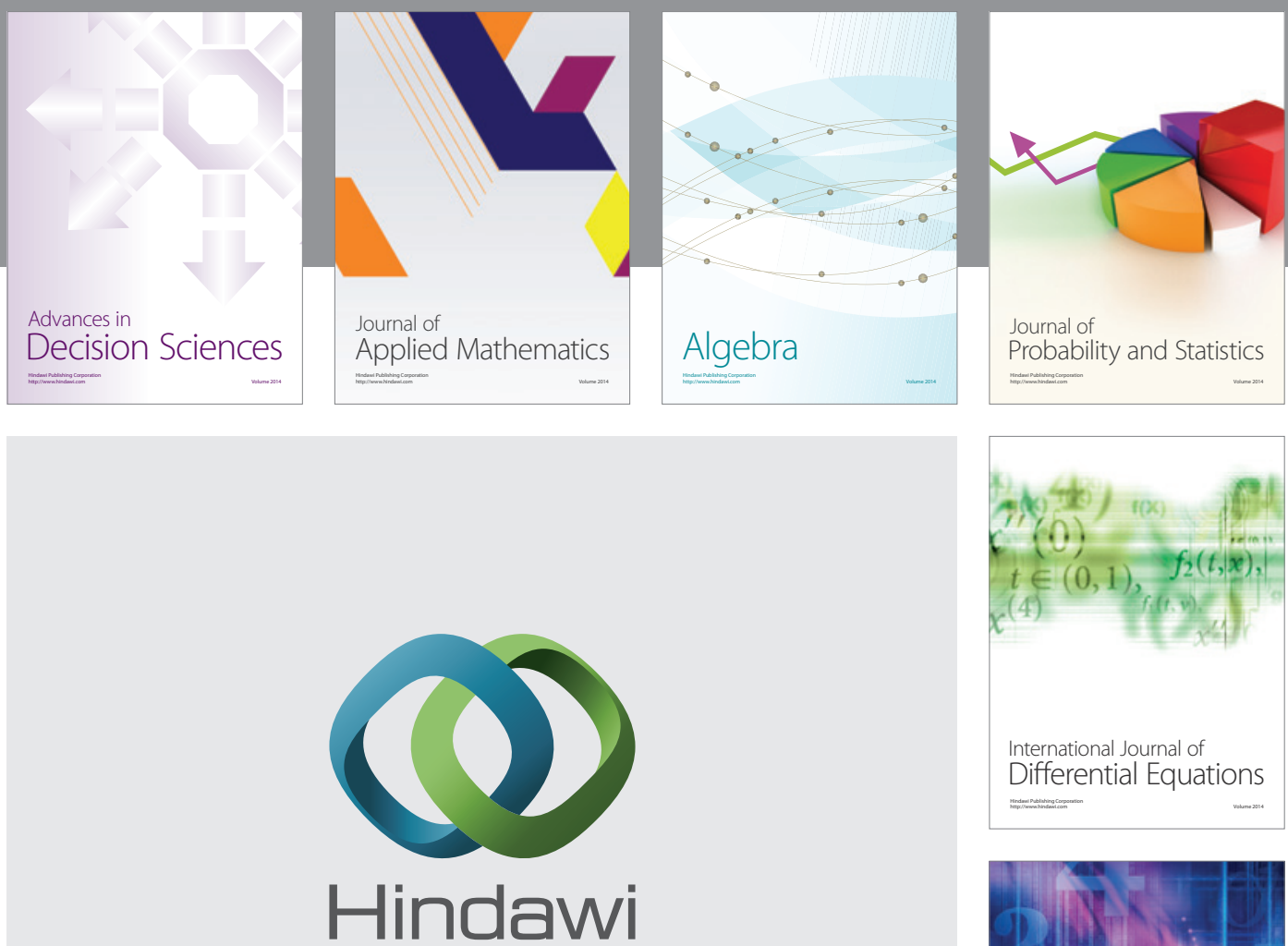

Submit your manuscripts at http://www.hindawi.com
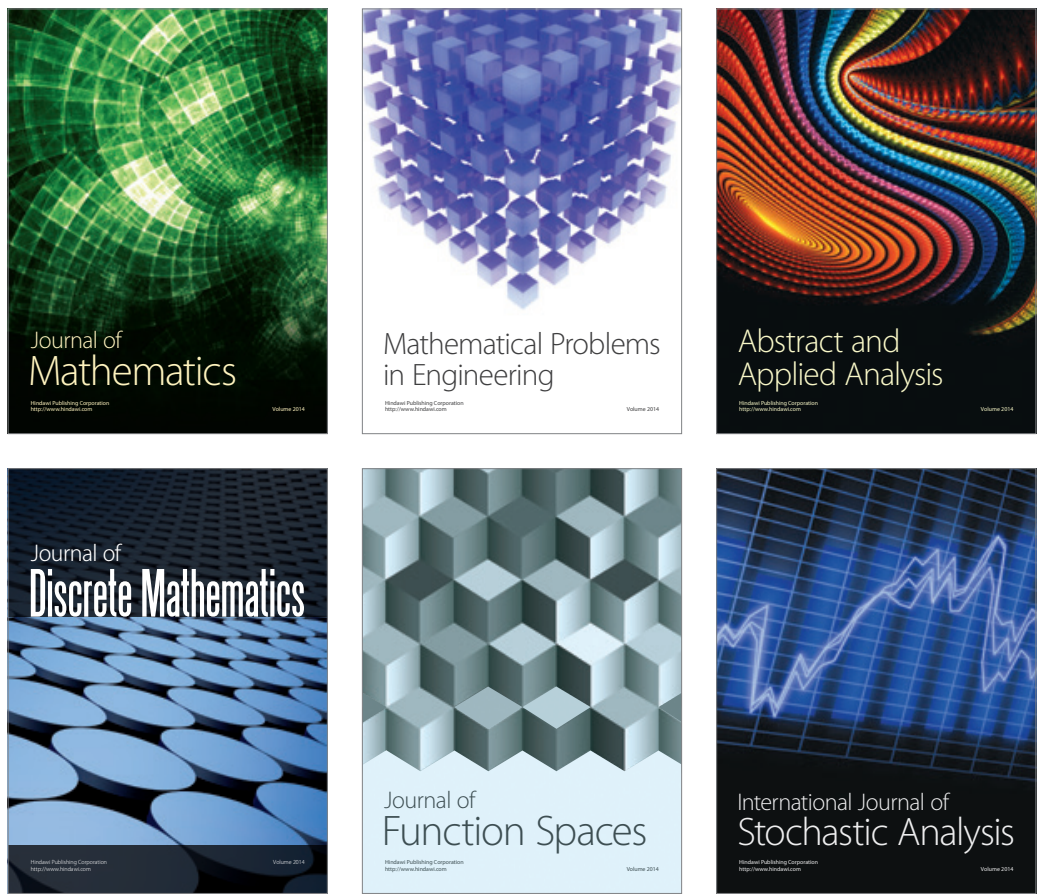

Journal of

Function Spaces

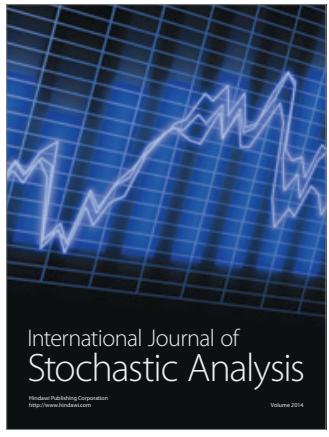

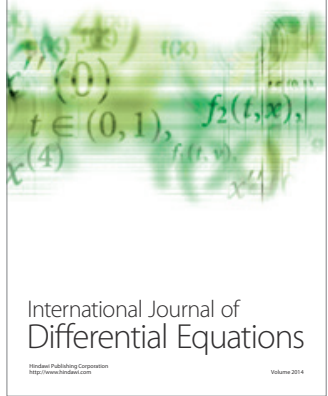
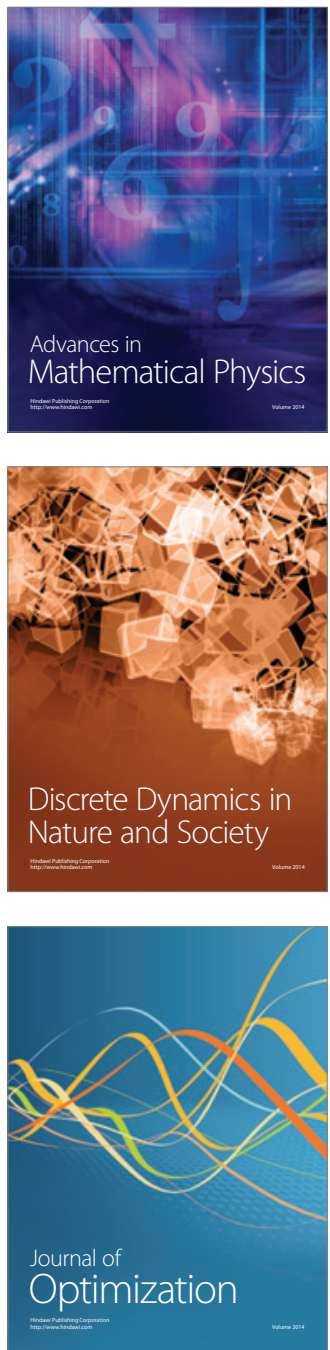